\title{
Homozygous PMS2 germline mutations in two families with early-onset haematological malignancy, brain tumours, HNPCC-associated tumours, and signs of neurofibromatosis type 1
}

\author{
Stefan Krüger*,1,5, Miriam Kinzel ${ }^{2}$, Constanze Walldorf ${ }^{3}$, Sven Gottschling ${ }^{4}$, Andrea Bier ${ }^{5}$, \\ Sigrid Tinschert ${ }^{5}$, Arend von Stackelberg ${ }^{6}$, Wolfram Henn ${ }^{7}$, Heike Görgens ${ }^{1}$, \\ Stephanie Boue ${ }^{8}$, Konrad Kölble ${ }^{9}$, Reinhard Büttner ${ }^{10}$ and Hans K Schackert ${ }^{1}$ \\ ${ }^{1}$ Department of Surgical Research, Dresden University of Technology, Dresden, Germany; ${ }^{2}$ Institute of Medical Genetics, \\ Charité Hospital, Humboldt University Berlin, Berlin, Germany; ${ }^{3}$ Institute of Human Genetics, University of Bonn, \\ Bonn, Germany; ${ }^{4}$ Department of Pediatric Haematology and Oncology, University Childreńs Hospital, Saarland \\ University Homburg, Homburg, Germany; ${ }^{5}$ Institute of Clinical Genetics, Dresden University of Technology, Dresden, \\ Germany; ${ }^{6}$ Department of Paediatric Oncology/Haematology, Charité Hospital, Humboldt University Berlin, Berlin, \\ Germany; ${ }^{7}$ Institute of Human Genetics, Saarland University Homburg, Homburg, Germany; ${ }^{8}$ Structural and \\ Computational Biology Unit, EMBL Heidelberg, Heidelberg, Germany; ${ }^{9}$ Institute of Pathology, Charité Hospital, \\ Humboldt University Berlin, Berlin, Germany; ${ }^{10}$ Institute of Pathology, University of Bonn, Bonn, Germany
}

Heterozygous germline mutations in mismatch repair (MMR) genes $M L H 1$, PMS2, MSH2, and MSH6 cause Lynch syndrome. New studies have indicated that biallelic mutations lead to a distinctive syndrome, childhood cancer syndrome (CCS), with haematological malignancies and tumours of brain and bowel early in childhood, often associated with signs of neurofibromatosis type 1 . We provide further evidence for CCS reporting on six children from two consanguineous families carrying homozygous PMS2 germline mutations. In family 1, all four children had the homozygous p.I590Xfs mutation. Two had a glioblastoma at the age of 6 years and one of them had three additional Lynch-syndrome associated tumours at 15. Another sibling suffered from a glioblastoma at age 9 , and the fourth sibling had infantile myofibromatosis at 1. In family 2, two of four siblings were homozygous for the p.G271V mutation. One had two colorectal cancers diagnosed at ages 13 and 14, the other had a Non-Hodgkin's lymphoma and a colorectal cancer at ages 10 and 11, respectively. All children with malignancies had multiple café-au-lait spots. After reviewing published cases of biallelic MMR gene mutations, we provide a concise description of CCS, revealing similarities in age distribution with carriers of heterozygous MMR gene mutations.

European Journal of Human Genetics (2008) 16, 62-72; doi:10.1038/sj.ejhg.5201923; published online 12 September 2007

Keywords: PMS2; homozygous mutations; childhood cancer syndrome (CCS); hereditary nonpolyposis colorectal cancer (HNPCC, Lynch) syndrome; mismatch repair (MMR) system

${ }^{*}$ Correspondence: Dr S Krüger, Institute of Clinical Genetics, Dresden University of Technology, Fetscherstraße 74, D-01307 Dresden, Germany. Tel: + 49 351/458 4277; Fax: + 49 351/458 4316;

E-mail: Stefan.Krueger@tu-dresden.de

Received 24 April 2007; revised 6 July 2007; accepted 16 August 2007; published online 12 September 2007

\section{Introduction}

Lynch syndrome (hereditary nonpolyposis colorectal cancer, HNPCC, MIM 114500), is the most common inherited cancer predisposition in humans alongside with hereditary breast and ovarian cancer syndrome, with an autosomal 
dominant mode of inheritance and a high penetrance. ${ }^{1}$ HNPCC is caused by heterozygous germline mutations in the DNA mismatch repair (MMR) genes MLH1, PMS2, MSH2, and $\mathrm{MSH}^{1}{ }^{1}$ and is characterised by highly microsatellite-unstable (MSI-H) tumours. ${ }^{2}$ Carriers of heterozygous MMR gene mutations are at high-risk for developing colorectal carcinomas (CRC), extracolonic neoplasias such as endometrial, small bowel, ureter and renal pelvis, stomach, ovarian, pancreas, biliary tract, and brain tumours, as well as sebaceous gland adenomas and keratoacanthomas. $^{3-5}$ Therefore, a specific surveillance programme for early detection of these cancers is recommended. ${ }^{6}$ Of the mutations in HNPCC 70-90\% occur in MLH1 and MSH2. ${ }^{7,8}$ The median age of disease onset (AO) of MLH1 and MSH2 mutation carriers is 40 and 43 years, respectively, with a wide range of AO. ${ }^{9} \mathrm{MSH} 6$ contributes to about $15 \%$ of all MMR gene mutations in HNPCC. The median AO in MSH6 mutation carriers is 51 years, thus, 10 years older than in $\mathrm{MLH} 1$ and $\mathrm{MSH} 2$ mutation carriers, also with a wide variation in $\mathrm{AO} .^{10}$ Until recently, it was uncertain if heterozygous PMS2 mutations contribute to HNPCC, yet lately several studies have provided evidence for the pathogenicity of heterozygous PMS2 germline mutations. ${ }^{11-14}$ Truninger et al $^{12}$ reported a median AO of 61.5 years in 16 patients with a loss of PMS2 expression in sporadic tumours. In a very recent study, a mean $\mathrm{AO}$ of 52 years for CRC and 54 years for endometrial cancer in patients meeting Bethesda criteria was reported. ${ }^{14}$ Furthermore, a lower penetrance of heterozygous PMS 2 mutations compared to the other three MMR genes has been suggested. ${ }^{12,15}$

In contrast to the well-known phenotype caused by heterozygous MMR gene mutations studied in thousands of families worldwide, little is known about the phenotype of the very rare biallelic MMR mutation carriers. Until recently, only 43 carriers with a biallelic MMR germline mutation from 23 different families have been reported. MLH1 was mutated in 7 families, ${ }^{16-22}$ MSH2 in 2 families, ${ }^{23,24}$ MSH6 in 4 families, ${ }^{25-28}$ and PMS2 in 10 families. ${ }^{15,29-33}$ Data published so far indicate that biallelic germline mutations in MMR genes cause a distinctive syndrome, characterised by haematological malignancies and tumours of the brain and the small and large bowel early in childhood, often associated with café-au-lait spots (CALS) and other signs of neurofibromatosis type 1 (NF1). This syndrome has been referred to as childhood cancer syndrome (CCS). ${ }^{32}$ Recently, the terms 'CoLoN' (for Colon tumours and/or leukaemia/lymphoma and/or neurofibromatosis features), ${ }^{34}$ and 'mismatch repair deficiency syndrome (MMR-D)' have been suggested. ${ }^{28}$

Hereby, we provide evidence for CCS in six children from two consanguineous families carrying homozygous PMS2 germline mutations. Five of them were affected by earlyonset malignancies from the tumour spectrum reported so far in biallelic MMR germline mutation carriers, and presented with multiple CALS. In addition, we report for the first time the occurrence of cancer of the ureter/renal pelvis at the age of 15 years, and an infantile myofibromatosis of the neck at 13 months of age in two children with homozygous PMS2 mutations. Furthermore, we summarise the clinical and molecular data from the published cases, providing a more concise description of the CCS phenotype.

\section{Materials and methods \\ Patient data}

Family 1 The male index patient (VII:2, Figure 1) suffered from glioblastoma multiforme (WHO grade IV) at 6 years of age. After surgical resection, he was treated with a combination of radiation and chemotherapy. At the age of 15, a lower gastrointestinal bleeding and the ensuing colonoscopy revealed 20 vulnerable polyps. Further examination showed three different simultaneous HNPCC-associated malignant tumours. Right-sided colon carcinoma in the caecum (T3 N1 GIII-IV), small bowel carcinoma in the jejunum (T4 NX GII), and carcinoma of the left urinary tract involving renal pelvis and upper part of the ureter (T3 NX GIII-IV). In addition, magnetic resonance imaging revealed a relapse of the glioblastoma multiforme. All three abdominal tumours were surgically removed. He died 13 months later due to progression of the unresectable brain tumour. He received a retinoic acid treatment in the tumour-free interval, which is published in detail elsewhere. ${ }^{35}$ His sister (VII:3) was diagnosed with glioblastoma multiforme at age of 6 years. After surgical resection, chemotherapy, and radiation she died at 7 years of age due to an unresectable relapse. Both children had multiple large CALS while lacking other features of NF1. A brother (VII:4) had three colonic polyps at the age of 8 years and was recently diagnosed with glioblastoma multiforme at the age of 9. Abdominal ultrasound examination was inconspicuous. Another brother of these siblings (VII:5) suffered from an infantile myofibromatosis of the neck at the age of 13 months, which was incurable despite of several attempts of surgical and chemotherapeutical treatments. He died at the age of 5 years. A paternal uncle (IV:3) of the siblings' father reportedly developed a malignant brain tumour at age of 10 years and died at the age of 13 years. The parents (VI:6 and VI:7) of the siblings are second cousins once removed. The coefficient of relationship was $0.0156(1 / 64)$ and the coefficient of inbreeding was 0.0078 $(1 / 128)$. They were 41 and 43 years old and clinically asymptomatic, but have refused to undergo surveillance programme so far.

Family 2 The female index patient (VII:10, Figure 2) presented firstly with a surgically removed (R0 resection) undifferentiated adenocarcinoma of the sigmoid colon at age of 13 years. As the tumour infiltrated the retro- 


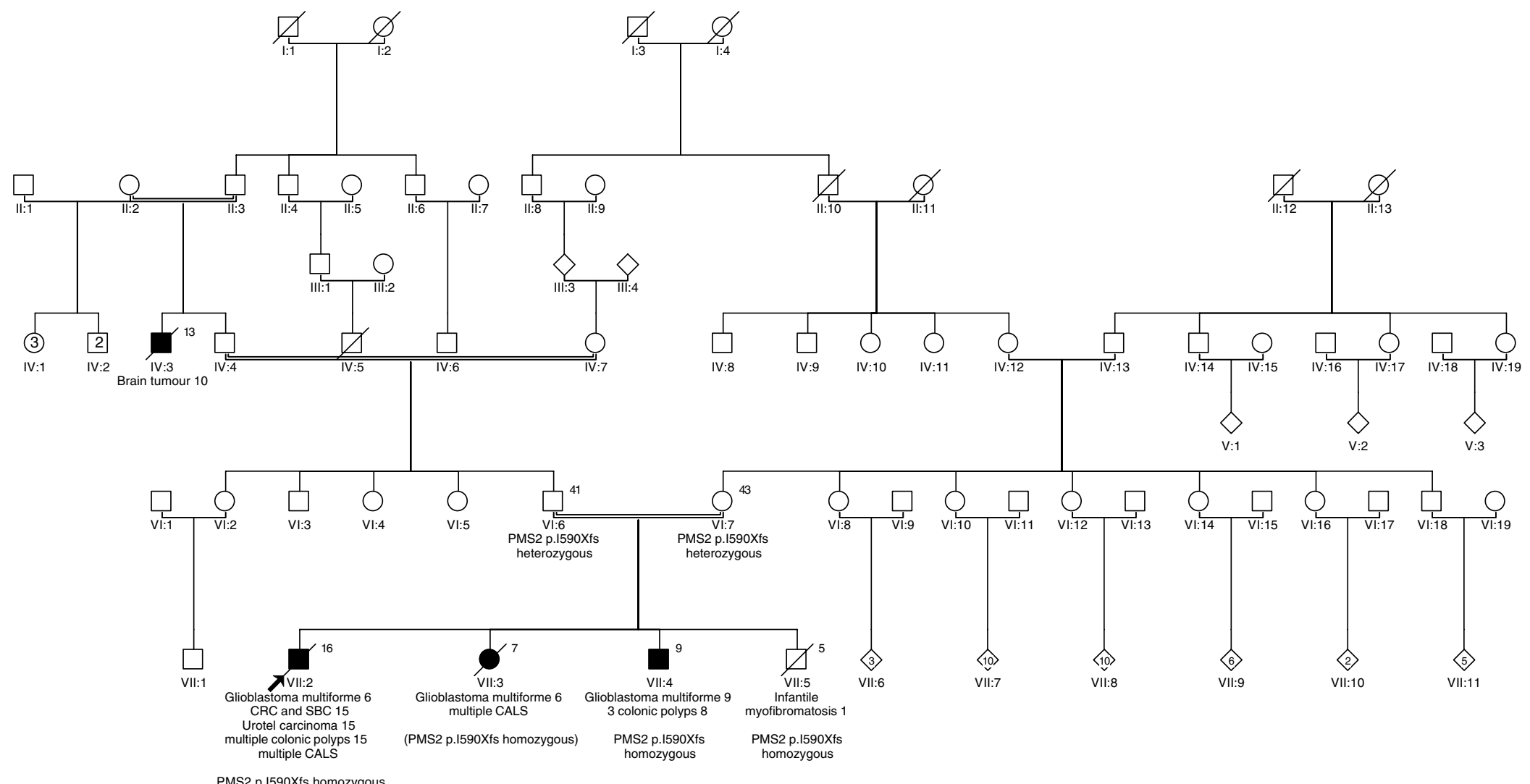

Figure 1 Pedigree of family 1. Tumours and age of onset are reported. Black symbols indicate CCS-related tumours (SBC, small bowel cancer; CRC, colorectal cancer). Numbers on right-top give the current age or age of death. The index patient is indicated by an arrow. In addition, signs of NF1 and mutational status are given (in parenthesis, inferred). 


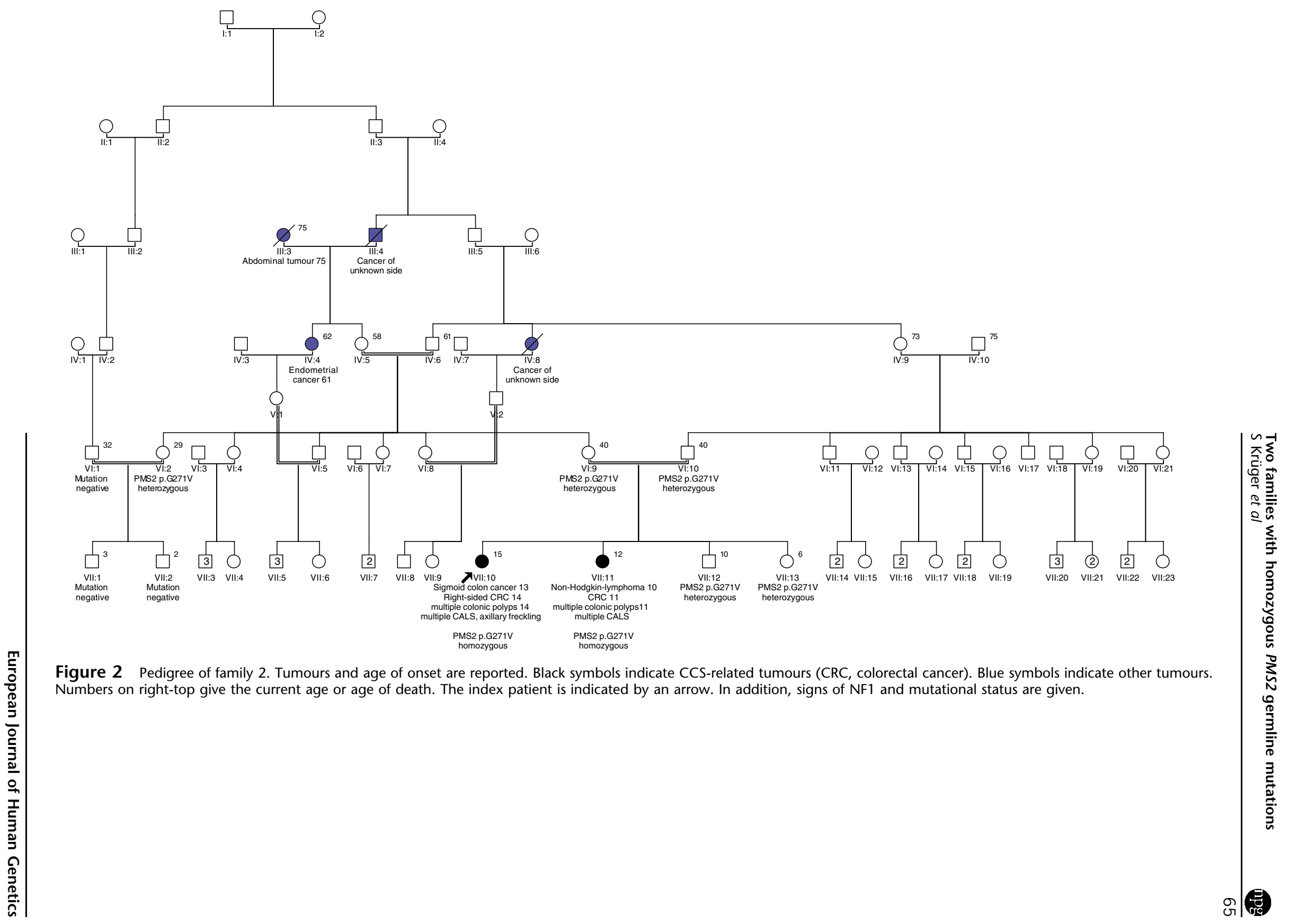


peritoneal space, and 14 out of 25 locoregional lymph nodes where involved, the disease was classified as stadium III (T4, N2, M0). She was treated according to the FOLFOX (5-FU, Folinic acid, Oxaliplatinum) regimen for 10 months. During the course of treatment, multiple colonic polyps were detected in a control colonoscopy, seven of which with high-grade dysplasia in the ascending colon, on the right flexure, in the transverse colon, and in the rectum. In addition, a right-sided CRC was diagnosed. The patient was treated by proctocolectomy of the remaining colon. Follow-up examinations did not reveal evidence of the malignant disease until now. The patient showed multiple CALS together with discrete axillary freckling (Figure 3ad), but no other signs of NF1 such as Lisch nodules or neurofibromas. The CALS in the right inguinal region $(20 \times 30 \mathrm{~mm}$ in size, Figure $3 \mathrm{a})$ had a sharply delineated appearance, with colour and shape typical of NF1. The CALS on the right upper arm $(20 \times 10 \mathrm{~mm}$ in size, Figure $3 \mathrm{~b}$ ) had more irregular margins, but still typical of NF1. In contrast, CALS on the dorsum $(15 \times 7$ and $8 \times 8 \mathrm{~mm}$ in size, Figure 3c) were more variable in size, shape, and pigmentation, and had irregular margins. Axillary freckling (Figure 3d) did not have a diffuse pattern
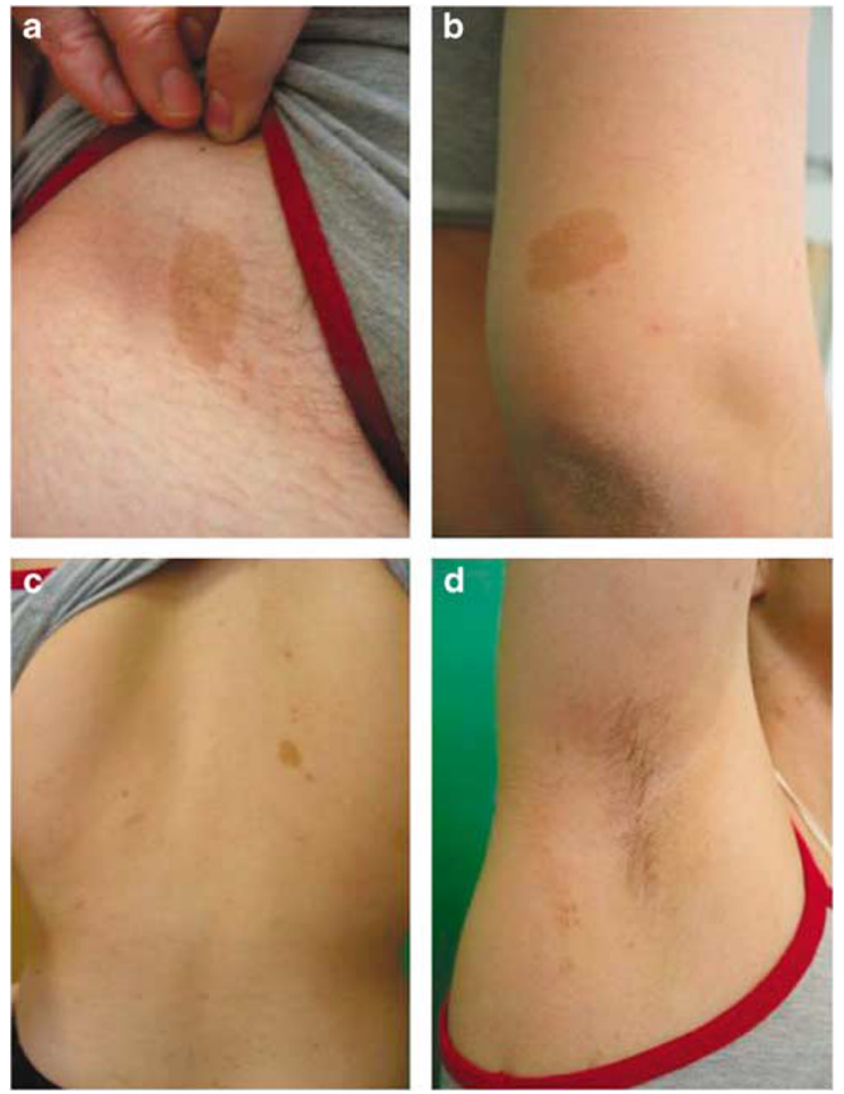

Figure 3 Signs of NF1 in the index patient of family 2. (a-c) CALS in the right inguinal region, right upper arm, and dorsum, respectively. (d) Freckling in the right axilla. like in NF1, but rather a more linear appearance, not excluding the possibility of segmental NF1. Her sister (VII:11) initially suffered from a T-cell Non-Hodgkin's lymphoma (NHL) at the age of 10 years, which was treated by chemotherapy according to the protocol Euro-LB 02 . Due to the CRCs of her sister, a colonoscopy was carried out at the age of 11, which identified 11 colonic polyps and 1 CRC. A proctocolectomy was performed. Follow-up examinations showed a complete remission of the NHL as well as the CRC until now. The patient had multiple CALS (left flank, $30 \times 40 \mathrm{~mm}$; dorsum, $20 \times 10 \mathrm{~mm}$; bottom $40 \times 30 \mathrm{~mm}$; right elbow, $40 \times 30 \mathrm{~mm}$ ) without other signs of NF1. A 10-year-old brother (VII:12) and a 6-year-old sister (VII:13) were healthy, yet there were some other malignancies in this family: the sister (IV:4) of the maternal grandmother of the index patient suffered from an endometrial cancer at the age of 61 years. Her mother (III:3) had an abdominal tumour at age of 75 and her father (III:4) had a cancer of unknown site. A sister (IV:8) of the maternal grandfather of the index patient who was also the sister of the paternal grandmother suffered from a cancer of unknown site. The parents (VI:9 and VI:10) of the index patient were first cousins. Furthermore, the parents (IV:5 and IV:6) of the mother were first cousins by themselves. Thus, the relationship coefficient of the parents of the index patients (VI:9 and VI:10) was 0.156 (1/8 plus $1 / 32)$ and the inbreeding coefficient of the index patient (VII:10) was 0.078 . Both parents were 40 years old and asymptomatic, but refused to have further examinations.

Both families were of Turkish origin living in Germany. All individuals investigated or, in case of children their parents, were given written informed consent for study participation. A total of 118 anonymous healthy blood donors from the Dresden Regional Blood Centre served as controls.

\section{Immunohistochemistry}

Immunohistochemical analyses were performed for MLH1, PMS2, MSH2, and MSH6 on 5- $\mu$ m-thick, formalin-fixed, paraffin-embedded sections from tumours of both index patients as described previously. ${ }^{36}$ Staining in the absence of the primary antibody served as negative controls. The normal staining pattern for all four antibodies was nuclear. Staining was evaluated by subjective assessment by at least two independent examiners.

\section{Molecular analyses}

Analysis for microsatellite instability (MSI) was performed on paired samples of leukocyte DNA and paraffinembedded tumour tissue as described previously. ${ }^{37}$ The markers of the reference panel, according to the international guidelines for the evaluation of MSI in CRC (BAT25, BAT26, D2S123, D5S346, D17S250), were applied. ${ }^{38}$ Tumours were classified as highly microsatellite unstable (MSI-H) if at least 30\% of the markers showed instabilities. 
Mutation analysis of MLH1, MSH2, and PMS2 was performed on genomic DNA isolated from peripheral blood leukocytes by amplifying and direct sequencing of the entire coding sequences and flanking intronic regions of these genes. Sequence analysis in MLH1 and MSH2 was carried out using primers and conditions published elsewhere, $^{39,40}$ using Thermo Sequenase Fluorescent Cycle Sequencing kit and Automated Laser Fluorescence (A.L.F.) express sequencing devices as described previously. ${ }^{36}$ Sequencing of PCR products of the PMS2 gene was performed using ABI PRISM ${ }^{\circledR}$ BigDye ${ }^{\circledR}$ Terminator Cycle Sequencing Ready Reaction Kit with AmpliTaq ${ }^{\circledR}$ DNA Polymerase v3.0 and v3.1 (Applied Biosystems). After an initial denaturation step at $95^{\circ} \mathrm{C}$ for $1 \mathrm{~min}$, amplification was performed using 30 cycles of denaturation $\left(96^{\circ} \mathrm{C}\right.$ for $10 \mathrm{~s})$, annealing $\left(52^{\circ} \mathrm{C}\right.$ for $\left.5 \mathrm{~s}\right)$, and extension $\left(60^{\circ} \mathrm{C}\right.$ for 3 min). Sequences were analysed with the ABI PRISM ${ }^{\mathbb{R}} \mathrm{AB}$ DNA Sequencing Analysis Software v5.1.1 (Applied Biosystems). To ensure the correct analysis of PMS2, we have used primers and conditions for PCR as described by De Vos et $a{ }^{32}$ which allow the specific amplification of the PMS2 exons while avoiding false co-amplification of one of the known PMS2 pseudogenes.

To assess the potential functional significance of the PMS2 missense mutation Gly271Val, we have applied two computational methods, sorting intolerant from tolerant amino acid substitutions (SIFT) and polymorphism phenotyping (polyphen). SIFT uses an evolutionary approach and is based on the assumption that important amino acids tend to be conserved across species. SIFT assigns a substitution probability from 0 to 1 for each possible amino-acid change. Substitutions with probabilities $<0.05$ are considered as intolerant (ie, functionally significant), whereas those $\geq 0.05$ are inferred as tolerated substitutions. ${ }^{41}$ Polyphen takes into account not only the evolutionary conservation of the amino acid subjected to the mutation, but also the physico-chemical characteristics of the wild-type and mutated amino-acid residue and the consequence of the amino acid change for the structural properties of the protein. ${ }^{42}$

Software package Cyrillic v.2.1.2 (Cherwell Scientific Publishing Limited, Oxford, UK) was used to calculate inbreeding coefficients.

\section{Results}

From the index patient in family 1 (VII:2, Figure 1), the right-sided CRC was analysed and showed a MSI-H phenotype with instabilities in three out of the five markers investigated by MSI analysis. The adenocarcinoma of the sigmoid colon from the index patient in family 2 (VII:10, Figure 2) was subjected to MSI analysis and was found to be also MSI-H. Immunohistochemistry revealed a complete loss of PMS2 expression along with normal expression of MLH1, MSH2, and MSH6 in both tumours mentioned above. This loss of PMS2 expression was not only found in the tumour cells, as usually seen in tumours of heterozygous MMR germline mutation carriers, but also in the adjacent non-neoplastic cells such as normal crypt cells or tumour-infiltrating lymphocytes (Figures $4 \mathrm{a}-\mathrm{d}$ and $5 \mathrm{a}-\mathrm{e})$. Staining with the same antibodies against the four MMR proteins and using the same reagents in specimens of other patients in the same immunohistochemical series showed normal staining of all four proteins, suggesting that the complete absence of PMS2 expression in tumour and non-neoplastic cells in the tumours of the index patients from families 1 and 2 was not an artefact (Figure 5f).

Complete mutation analysis in the MMR genes MLH1, MSH2, and PMS2 was performed in both index patients. Analyses of MLH1 and MSH2 did not show a pathogenic mutation neither in the index patient from family 1 nor in the index patient from family 2 .

In contrast, sequencing of $P M S 2$ revealed a deletion of an adenine in exon 11 (c.1768delA) in the index patient of family 1 . This deletion led to a premature stop codon four triplets downstream, predicted to result in a truncated protein (p.I590fsX594). This mutation was found in a homozygous state and was confirmed by sequencing the opposite DNA strand. Both parents (VI:6 and VI:7, Figure 1) were heterozygous for the mutation found in the index patient, whereas both brothers of the index patient (VII:4 and VII:5, Figure 1) were homozygous carriers of this mutation. Analysis of a 10-year-old paraffin-embedded tissue DNA of the deceased sister of the index patient (VII:3, Figure 1) was not successful because of poor DNA quality, yet based on the phenotype the existence of the mutation in a homozygous state can be inferred.

In the index patient of family 2, a homozygous transversion from guanine to thymine was detected in exon 8 of PMS2 (c.812G $>$ T) and confirmed by sequencing the opposite DNA strand. This transversion led to an amino acid change from glycine to valine at codon 271 (p.G271V). This missense mutation was not found in all 236 alleles from 118 healthy control individuals screened. SIFT analysis with inclusion of the human PMS2 sequence and 37 closely related protein sequences from various species gave a substitution probability of 0.01 , predicting that this amino-acid change is not tolerated (ie, functionally significant). Polyphen tests also predicted that the mutation Gly271Val was deleterious. Firstly, this position is extremely well conserved during evolution (emphasised by the high polyphen score). Moreover, the amino-acid change by itself is drastic due to the mutation from a small residue into a large one, and the mutation is located in a seemingly branching point in the structure, which might be involved in the interaction with other proteins, or the mutation into a larger residue could disturb the overall 3D structure of the protein. 

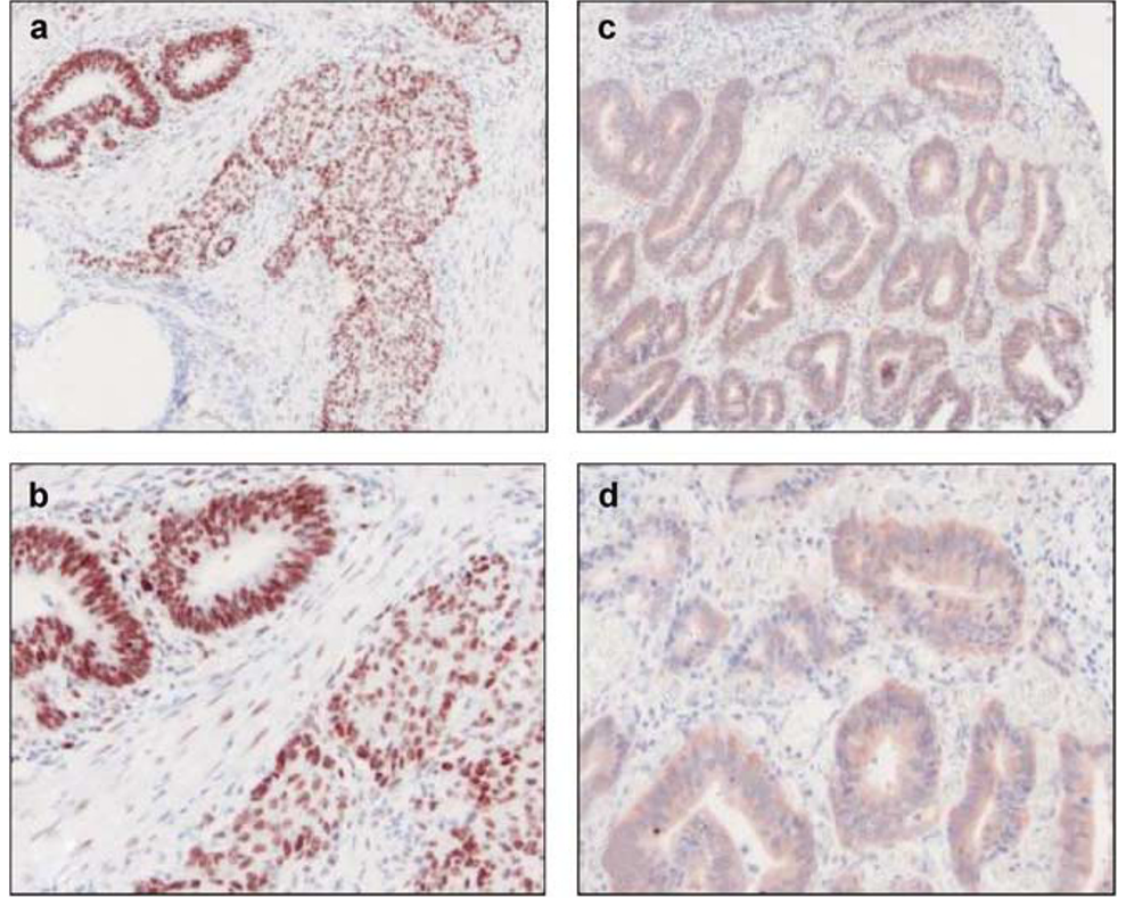

Figure 4 Expression of MMR proteins, analysed by immunohistochemistry. (a-d) Tumour sections of the caecal colon cancer of the index patient of family 1 harbouring biallelic PMS2 germline mutation p.590Xfs, (a and $\mathbf{b})$ immunostained with MLH1 antibodies. Normal nuclear staining in tumour cells and non-neoplastic cells. (c and d) Immunostaining with PMS2 antibody revealed complete loss of expression in tumour cells as well as in adjacent normal crypt cells and infiltrating lymphocytes.

Both parents (VI:9 and VI:10, Figure 2) and both healthy siblings (VII:12 and VII:13, Figure 2) of the index patient in family 2 were heterozygous for this mutation, whereas the affected sister (VII:11, Figure 2) was homozygous as the index patient herself. Furthermore, four other more distantly related, healthy relatives from family 2 were tested for this mutation (VI:1, VI:2, VII:1, VII:2). One of these (VI:2) was a heterozygous mutation carrier, the other three did not carry this mutation (Figure 2).

\section{Discussion}

We have identified two Turkish families living in Germany, in which six of eight children from consanguineous healthy heterozygous parents were homozygous carriers of a PMS2 mutation, five of which were proven and one inferred. Five of these children were affected by haematological malignancy, brain and/or intestinal tumours, and one suffered from an aggressive infantile myofibromatosis. Tumours from both index patients showed MSI-H and a complete loss of PMS2 expression along with normal expression of MLH1, MSH2, and MSH6. The loss of PMS2 expression was not only found in the tumour cells, but also in the adjacent non-neoplastic cells such as normal crypt cells or tumour-infiltrating lymphocytes (Figures $4 \mathrm{a}-\mathrm{d}$ and 5a-e). The absence of PMS2 expression in non-neoplastic cells is consistent with a knockout of the PMS2 gene caused by biallelic germline mutations. Furthermore, all children affected by malignancies showed cutaneous signs similiar to NF1, particularly CALS. The appearance of two CALS in the right inguinal region and on the right upper arm in the index patient of family 2 (Figure $3 a$ and $b$ ) were typical of NF1, in contrast to the more ragged-edged and slightly diffuse CALS on the dorsum (Figure 3c), which were more similar with those described by De Vos et $a l^{15}$ in patients with biallelic PMS2 mutations. Haematological malignancies, brain and intestinal tumours together with signs of NF1 are typical for a distinctive syndrome, referred to as CCS, which is caused by biallelic MMR gene germline mutations. ${ }^{32}$ In addition, one child suffered from a ureter/ renal pelvis carcinoma at the age of 15 years. Urothelial carcinomas are rare in general, yet are highly sensitive for HNPCC (alongside with CRC, endometrial, small bowel carcinomas) as defined in the Amsterdam II criteria. ${ }^{43}$ Nonetheless, urothelial carcinomas have not yet been reported in biallelic MMR gene mutation carriers. The only published extracolonic HNPCC-associated cancers in CCS were an endometrial and an ovarian cancer at the age of 23 years, also in a biallelic PMS2 mutation carrier. ${ }^{31}$ Thus, extracolonic HNPCC-associated tumours in biallelic MMR mutation carriers seem to occur less frequently and later in life. This resembles the situation in heterozygous mutation carriers. 

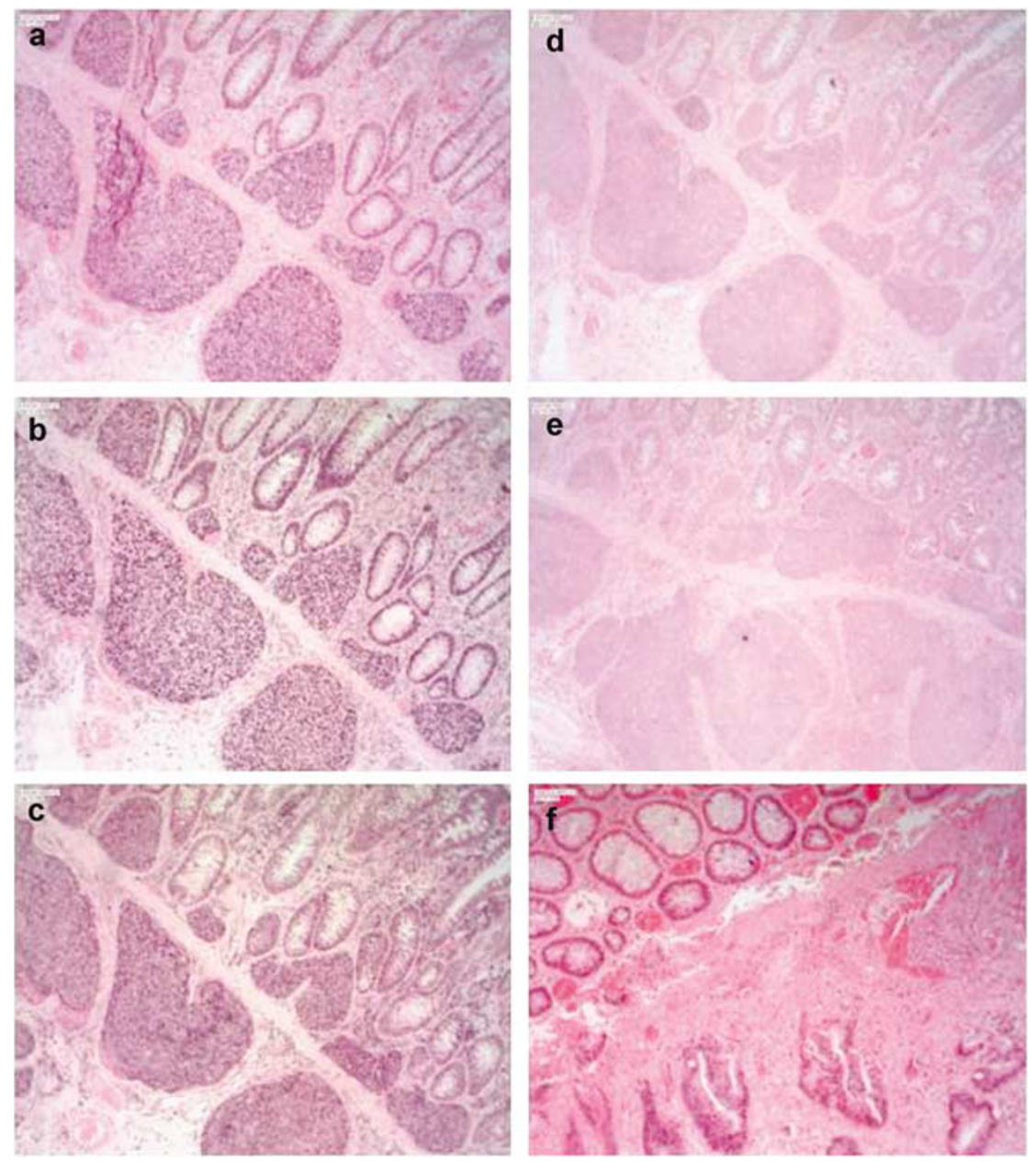

Figure 5 Expression of MMR proteins, analysed by immunohistochemistry. (a-e) Tumour sections of the sigmoid colon cancer of the index patient of family 2 harbouring biallelic PMS2 germline mutation p.G271V. (a-c) Immunostained with MLH1, MSH2, and MSH6 antibodies, respectively. Normal nuclear staining in tumour cells and non-neoplastic cells. (d and e) Immunostaining with PMS2 antibody showed complete loss of expression in tumour cells as well as in adjacent normal crypt cells and infiltrating lymphocytes. (f) Staining of tumour specimen of another patient with the same PMS2 antibody and reagents in the same immunohistochemical series showed normal staining and served as positive control.

While the pathogenicity of the truncating mutation p.I590Xfs found in family 1 is unquestionable, the interpretation of missense mutations such as p.G271V found in family 2 is difficult. Nevertheless, the pathogenicity of this mutation is strongly suggested by several aspects. Firstly, the mutation is located in the wellconserved N-terminal part of PMS2 (NhPMS2), which is active both in ATP hydrolysis and DNA binding. The structure of NhPMS2 consists of two $\alpha / \beta$ domains. The second domain (residues 239-365) consists of three helices and a five-stranded mixed $\beta$-sheet $(\beta 9-\beta 12)$. The aminoacid residue Gly271 maps to the second of these $\beta$-sheets $(\beta 10),{ }^{44}$ thus being possibly important for the NhPMS2 structure. In contrast to yeast, functional ATP hydrolysis is required for the formation of the MMR initiation complex in humans. ${ }^{45}$ Secondly, p.G271V was absent in 236 chromosomes from 118 healthy control individuals.
Thirdly, SIFT analysis indicated that this amino-acid change is intolerant (ie, functionally significant). Fourthly, polyphen analyses predicted that the mutation p.G271V is deleterious. Fifthly, the mutation co-segregated with the CCS phenotype in family 2. It was found only in the two children with early onset malignancies and CALS in a homozygous stage. Finally, immunohistochemistry clearly indicated a biallelic PMS2 germline mutation through complete loss of protein expression.

Consanguinity was reported in 12 of the 23 families so far published. The Turkish population comprises the largest group of foreigners living in Germany, around $26 \%$ of the total (German ministry of the interior, http://www.bmi. bund.de). In Turkey, the overall rate of consanguineous marriages is about $21 \%$, with an average inbreeding coefficient of $0.0065 .{ }^{46}$ Nevertheless, only one of the families with biallelic MMR gene mutations reported so 
far was of Turkish origin. The mutated gene in this family was MLH1. ${ }^{17}$ From the 10 known families with biallelic PMS2 mutations, three were heterozygous without consanguinity and came from Italy ${ }^{30,33}$ and Canada. ${ }^{29,32}$ In the remaining seven families, the affected children were homozygous and originated from consanguineous parents. One family from Guyana was reported, ${ }^{31}$ the other six came originally from Pakistan. ${ }^{15,32}$ Thus, the two families reported here were the first of Turkish origin with biallelic germline mutations in PMS2.

An extensive review of the literature revealed 43 individuals with biallelic MMR germline mutations in 23 different families affected with 55 haematological malignancies, brain or intestinal tumours. ${ }^{15-33}$ Two at least in part own studies were not considered, as the authors accentuated that these cases or not typical for CCS. ${ }^{47,48}$ Other tumours were rarely reported: one Wilms tumour, one neuroectodermal ovarian carcinoma, one ovarian adenocarcinoma, and one endometrial cancer. ${ }^{19,31}$ Altogether, including the cases reported in this study, 64 malignancies within the CCS spectrum have arisen in 49 individuals with biallelic MMR germline mutations in 25 families. The median AO was 9 years ranging from 1 to 24 years. Brain tumours occurred in most families, followed by haematological malignancies and intestinal tumours. The lowest $\mathrm{AO}$ was seen in haematological malignancies, followed by brain tumours and intestinal tumours. Extraintestinal HNPCC-associated tumours are rare and occur at an older age (Table 1). Thus, the CCS phenotype resembles the phenotype of MMR-knockout-mouse models, which have a severely reduced lifespan and develop primarily lymphomas at an early age and intestinal tumours later in life. ${ }^{49}$

The frequencies of biallelic MLH1, PMS2, MSH2, or MSH6 mutations in CCS families are different from those in Lynch syndrome families carrying heterozygous MMR gene mutations, most of which are MLH1 or MSH2 mutations. ${ }^{7,8}$ At least one reason for the unexpected high contribution of biallelic PMS 2 mutations to CCS (12 out of the 25 families, $48.0 \%$ ) is the existence of a founder mutation originating in the Mirpur region of northeastern Pakistan (p.R802X), identified in five families. Four of the five families had identical genotypes, suggesting an ancestral founder effect. ${ }^{15}$ Another explanation could be an underestimation of PMS2 mutations biased by the age restriction of the Bethesda guidelines as suggested by Truninger et al, ${ }^{12}$ who found PMS2 mutations in 1048 unselected, consecutive colorectal cancer patients in the same frequency as MSH2 and MLH1 mutations.

Notably, the lowest median AO was seen in biallelic MSH2 and MLH1 mutation carriers, followed by MSH6 and PMS2 mutation carriers. This mirrors the AO distribution of heterozygous MMR mutation carriers in Lynch syndrome patients, only 35 years earlier (Table 2). An explanation for the stronger phenotype and the younger $\mathrm{AO}$ in hetero-
Table 1 Occurrence of CCS malignancies, median AO, and range in 25 families with biallelic MMR germline mutations to date

\begin{tabular}{|c|c|c|c|}
\hline Site of malignancy & $\begin{array}{l}\text { Affected families (\% } \\
\text { of all families) }\end{array}$ & $\begin{array}{l}\text { Median } \\
\text { AO }\end{array}$ & Range \\
\hline $\begin{array}{l}\text { Haematological } \\
\text { malignancies }\end{array}$ & $15(60.0)$ & 5 & $1-17$ \\
\hline Brain tumours & $17(68.0)$ & 7 & $2-24$ \\
\hline Intestinal tumours & $12(48.0)$ & 13 & $8-22$ \\
\hline $\begin{array}{l}\text { Extraintestinal HNPCC- } \\
\text { associated tumours }\end{array}$ & $2(8.0)$ & 23 & $15-23$ \\
\hline
\end{tabular}

Table 2 Number of affected families and individuals, number of tumours, and median $\mathrm{AO}$ in 25 families with biallelic MMR germline mutations

\begin{tabular}{lcrcc}
\hline $\begin{array}{l}\text { Affected } \\
\text { gene }\end{array}$ & $\begin{array}{c}\text { Affected families } \\
\text { (individuals) }\end{array}$ & $\begin{array}{c}\text { Median } \\
\text { AO }\end{array}$ & Range & $\begin{array}{c}\text { Number of } \\
\text { tumours }\end{array}$ \\
\hline MSH2 & $2(3)$ & 2 & $1-3$ & 3 \\
MLH1 & $7(12)$ & 4 & $1-22$ & 11 \\
MSH6 & $4(6)$ & 9 & $3-13$ & 12 \\
PMS2 & $12(28)$ & 12 & $2-24$ & 38 \\
\hline
\end{tabular}

zygous MSH2 and MLH1 mutation carriers could be the complete loss of MutS and MutL function, respectively, as MLH1 and MSH2 protein did not have an alternate binding partner to form heterodimers. In case of loss of MSH6 protein, MSH2 can alternatively bind to MSH3 (MutS $\beta$ ) and, in case of loss of PMS2 protein, MLH1 can alternatively bind to PMS1 (MutL $\beta$ ) or MLH3 (MutL $\gamma$ ), which may explain the lower penetrance of MSH6 and PMS2 mutations. The same mechanism could explain the observation in biallelic MMR mutation carriers.

Family history of malignancies was evident in 13 of 25 families (52.0\%) comprising 7 families with a classical Lynch syndrome and a clustering of malignancies in the remaining 6 families. The absence of family history of malignancies in the remaining families is surprising, as all parents of affected children with biallelic MMR mutations must be assumed as heterozygous mutation carriers. It is possible that some of the parents were still too young to develop Lynch syndrome. Remarkably, the absence of family history was most frequent in families with biallellic PMS2 mutations. None of the 12 families had a Lynch syndrome characteristic, and only in 4 families clustering of malignancies occurred. This might be due to a higher AO and a lower penetrance of heterozygous PMS2 mutations.

Homozygous MMR gene mutations were identified in 18 families, and consanguinity was reported in 14 of these families. Seven families carried compound heterozygous biallelic MMR gene mutations.

Biallelic MMR gene mutation carriers from 21 families had signs of NF1, mostly multiple CALS, but seldom 
fulfilled the NIH diagnostic criteria for NF1. ${ }^{50,51}$ None of the parents of biallelic MMR gene mutation carriers showed NF1 signs, which substantiates the hypothesis, that the NF1 gene is an early target in embryogenesis of carriers of biallelic MMR gene mutations. This is further supported by a higher rate of NF1 gene mutations in highly unstable human cell lines and tumours as well as in Mlh1 knockout mice compared to MMR-sufficient tissue. ${ }^{52} \mathrm{~A}$ somatic inactivation of NF1 gene would lead to mosaicism, which would result in a segmental NF1 phenotype as seen in patient VII:10 of family 2.

Therefore, the family history does not seem to be a good predictor for identifying CCS, while the early onset of haematological malignancies, brain or intestinal tumours together with signs of NF1 identified CCS in 20 out of the 25 families (80.0\%) with biallelic MMR gene germline mutation carriers.

In conclusion, the two families hereby presented further enhance CCS as a distinctive syndrome with typical tumour spectrum, an early AO and often accompanied by signs of NF1, which is caused by biallelic germline mutations in one of the MMR genes. CCS should be considered in cases of HNPCC-associated tumours, brain tumours, or haematological malignancies at an unusual young age in association with signs of NF1, particularly when the parents are consanguine. Additionally, the summary of the phenotypes of all known biallelic MMR gene mutation carriers revealed that the differences between single MMR genes in CCS (ie, in homozygous or compound heterozygous mutational state) seem to resemble the differences between single MMR genes known from Lynch syndrome (ie, in heterozygous mutational state).

\section{Acknowledgements}

We thank Ms M Reichmann, Ms S Steiner, and Mr A Schiewart for excellent technical assistance. This work was supported by the Verbundprojekt 'Familiärer Darmkrebs' of the Deutsche Krebshilfe (DKH, German Cancer Aid, 70-3032).

\section{References}

1 de la Chapelle A: Genetic predisposition to colorectal cancer. Nat Rev Cancer 2004; 4: 769-780.

2 Ionov Y, Peinado MA, Malkhosyan S, Shibata D, Perucho M: Ubiquitous somatic mutations in simple repeated sequences reveal a new mechanism for colonic carcinogenesis. Nature 1993; 363: 558-561.

3 Lynch HT, Smyrk TC, Watson P et al: Genetics, natural history, tumour spectrum, and pathology of hereditary nonpolyposis colorectal cancer: an updated review. Gastroenterology 1993; 104: $1535-1549$.

4 Vasen HF, Wijnen JT, Menko FH et al: Cancer risk in families with hereditary nonpolyposis colorectal cancer diagnosed by mutation analysis. Gastroenterology 1996; 110: 1020-1027.

5 Umar A, Boland CR, Terdiman JP et al: Revised Bethesda Guidelines for hereditary nonpolyposis colorectal cancer (Lynch syndrome) and microsatellite instability. J Natl Cancer Inst 2004; 96: $261-268$.
6 Dunlop MG: British Society for Gastroenterology; Association of Coloproctology for Great Britain and Ireland. Guidance on gastrointestinal surveillance for hereditary non-polyposis colorectal cancer, familial adenomatous polypolis, juvenile polyposis, and Peutz-Jeghers syndrome. Gut 2002; 51: V21-V27.

7 Liu B, Parsons R, Papadopoulos N et al: Analysis of mismatch repair genes in hereditary non-polyposis colorectal cancer patients. Nat Med 1996; 2: 169-174.

8 Peltomaki P: Deficient DNA mismatch repair: a common etiologic factor for colon cancer. Hum Mol Genet 2001; 10: 735-740.

9 Goecke T, Schulmann K, Engel C et al: Genotype-phenotype comparison of German MLH1 and MSH2 mutation carriers clinically affected with Lynch syndrome: a report by the German HNPCC Consortium. J Clin Oncol 2006; 24: 4285-4292.

10 Plaschke J, Engel C, Kruger S et al: Lower incidence of colorectal cancer and later age of disease onset in 27 families with pathogenic MSH6 germline mutations compared to families with MLH1 or MSH2 mutations. J Clin Oncol 2004; 22: 4486-4494.

11 Nakagawa H, Lockman JC, Frankel WL et al: Mismatch repair gene PMS2: disease-causing germline mutations are frequent in patients whose tumors stain negative for PMS2 protein, but paralogous genes obscure mutation detection and interpretation. Cancer Res 2004; 64: 4721-4727.

12 Truninger $\mathrm{K}$, Menigatti $\mathrm{M}$, Luz J et al: Immunohistochemical analysis reveals high frequency of PMS2 defects in colorectal cancer. Gastroenterology 2005; 128: 1160-1171.

13 Worthley DL, Walsh MD, Barker M et al: Familial mutations in PMS2 can cause autosomal dominant hereditary nonpolyposis colorectal cancer. Gastroenterology 2005; 128: 1431-1436.

14 Hendriks YM, Jagmohan-Changur S, van der Klift HM et al: Heterozygous mutations in PMS2 cause hereditary nonpolyposis colorectal carcinoma (Lynch syndrome). Gastroenterology 2006; 130: $312-322$.

15 De Vos M, Hayward BE, Charlton R et al: PMS2 mutations in childhood cancer. J Natl Cancer Inst 2006; 98: 358-361.

16 Wang Q, Lasset C, Desseigne F et al: Neurofibromatosis and early onset of cancers in hMLH1-deficient children. Cancer Res 1999; 59: 294-297.

17 Ricciardone MD, Ozcelik T, Cevher B et al: Human MLH1 deficiency predisposes to haematological malignancy and neurofibromatosis type 1. Cancer Res 1999; 59: 290-293.

18 Vilkki S, Tsao JL, Loukola A et al: Extensive somatic microsatellite mutations in normal human tissue. Cancer Res 2001; 61: 45414544.

19 Wagner A, Reddingius $\mathrm{R}$, Kros $\mathrm{J}$ et al: Wilms tumor and glioblastoma in a child with double MLH1 germline mutation. Fam Cancer 2003; 2: 57.

20 Gallinger S, Aronson M, Shayan K et al: Gastrointestinal cancers and neurofibromatosis type 1 features in children with a germline homozygous MLH1 mutation. Gastroenterology 2004; 126: 576-585.

21 Raevaara TE, Gerdes AM, Lonnqvist KE et al: HNPCC mutation MLH1 P648S makes the functional protein unstable, and homozygosity predisposes to mild neurofibromatosis type 1 . Genes Chromosomes Cancer 2004; 40: 261-265.

22 Rey JM, Noruzinia M, Brouillet JP, Sarda P, Maudelonde T, Pujol P: Six novel heterozygous MLH1, MSH2, and MSH6 and one homozygous MLH1 germline mutations in hereditary nonpolyposis colorectal cancer. Cancer Genet Cytogenet 2004; 155: $149-151$.

23 Whiteside D, McLeod R, Graham G et al: A homozygous germline mutation in the human $\mathrm{MSH} 2$ gene predisposes to haematological malignancy and multiple cafe-au-lait spots. Cancer Res 2002; 62: 359-362.

24 Bougeard G, Charbonnier F, Moerman A et al: Early onset brain tumor and lymphoma in MSH2-deficient children. Am J Hum Genet 2003; 72: 213-216.

25 Hegde MR, Chong B, Blazo ME et al: A homozygous mutation in MSH6 causes Turcot syndrome. Clin Cancer Res 2005; 11: $4689-4693$. 
26 Menko FH, Kaspers GL, Meijer GA, Claes K, van Hagen JM, Gille JJ: A homozygous MSH6 mutation in a child with cafe-au-lait spots, oligodendroglioma and rectal cancer. Fam Cancer 2004; 3 : $123-127$.

27 Ostergaard JR, Sunde L, Okkels H: Neurofibromatosis von Recklinghausen type I phenotype and early onset of cancers in siblings compound heterozygous for mutations in MSH6. Am J Med Genet A 2005; 139: 96-105.

28 Scott RH, Mansour S, Pritchard-Jones K, Kumar D, MacSweeney F, Rahman N: Medulloblastoma, acute myelocytic leukemia and colonic carcinomas in a child with biallelic MSH6 mutations. Nat Clin Pract Oncol 2007; 4: 130-134.

29 Hamilton SR, Liu B, Parsons RE et al: The molecular basis of Turcot's syndrome. N Engl J Med 1995; 332: 839-847.

30 de Rosa M, Fasano C, Panariello L et al: Evidence for a recessive inheritance of Turcot's syndrome caused by compound heterozygous mutations within the PMS2 gene. Oncogene 2000; 19: $1719-1723$.

31 Trimbath JD, Petersen GM, Erdman SH, Ferre M, Luce MC, Giardiello FM: Cafe-au-lait spots and early onset colorectal neoplasia: a variant of HNPCC? Fam Cancer 2001; 1: 101-105.

32 De Vos M, Hayward BE, Picton S, Sheridan E, Bonthron DT: Novel PMS2 pseudogenes can conceal recessive mutations causing a distinctive childhood cancer syndrome. Am J Hum Genet 2004; 74: 954-964.

33 Agostini M, Tibiletti MG, Lucci-Cordisco E et al: Two PMS2 mutations in a Turcot syndrome family with small bowel cancers. Am J Gastroenterol 2005; 100: 1886-1891.

34 Bandipalliam P: Syndrome of early onset colon cancers, hematologic malignancies \& features of neurofibromatosis in HNPCC families with homozygous mismatch repair gene mutations. Fam Cancer 2005; 4: 323-333.

35 Gottschling S, Reinhard H, Pagenstecher C et al: Hypothesis: possible role of retinoic acid therapy in patients with biallelic mismatch repair gene defects. Eur J Pediatr 2007; E-pub ahead of print.

36 Kruger S, Plaschke J, Jeske B et al: Identification of six novel MSH2 and MLH1 germline mutations in HNPCC. Hum Mutat 2003; 21: $445-446$

37 Plaschke J, Kruger S, Pistorius S, Theissig F, Saeger HD, Schackert HK: Involvement of hMSH6 in the development of hereditary and sporadic colorectal cancer revealed by immunostaining is based on germline mutations, but rarely on somatic inactivation. Int J Cancer 2002; 97: 643-648.

38 Boland CR, Thibodeau SN, Hamilton SR et al: A National Cancer Institute Workshop on Microsatellite Instability for cancer detection and familial predisposition: development of inter- national criteria for the determination of microsatellite instability in colorectal cancer. Cancer Res 1998; 58: 5248-5257.

39 Kolodner RD, Hall NR, Lipford J et al: Structure of the human MLH1 locus and analysis of a large hereditary nonpolyposis colorectal carcinoma kindred for mlhl mutations. Cancer Res 1995; 55: 242-248

40 Kolodner RD, Hall NR, Lipford J et al: Structure of the human MSH2 locus and analysis of two Muir-Torre kindreds for MSH2 mutations. Genomics 1994; 24: 516-526.

$41 \mathrm{Ng}$ PC, Henikoff S: Accounting for human polymorphisms predicted to affect protein function. Genome Res 2002; 12: 436-446.

42 Ramensky V, Bork P, Sunyaev S: Human non-synonymous SNPs: server and survey. Nucleic Acids Res 2002; 30: 3894-3900.

43 Vasen HF, Watson P, Mecklin JP, Lynch H: New clinical criteria for hereditary nonpolyposis colorectal cancer (HNPCC, Lynch syndrome) proposed by the International Collaborative group on HNPCC. Gastroenterology 1999; 116: 1453-1456.

44 Guarne A, Junop MS, Yang W: Structure and function of the $\mathrm{N}$-terminal $40 \mathrm{kDa}$ fragment of human PMS2: a monomeric GHL ATPase. EMBO J 2001; 20: 5521-5531.

$45 \mathrm{Gu}$ L, Hong Y, McCulloch S, Watanabe H, Li GM: ATP-dependent interaction of human mismatch repair proteins and dual role of PCNA in mismatch repair. Nucleic Acids Res 1998; 26: $1173-1178$

46 Basaran N, Sayli BS, Basaran A, Solak M, Artan S, Stevenson JD: Consanguineous marriages in the Turkish population. Clin Genet 1988; 34: 339-341.

47 Plaschke J, Linnebacher M, Kloor $\mathrm{M}$ et al: Compound heterozygosity for two MSH6 mutations in a patient with early onset of HNPCC-associated cancers, but without hematological malignancy and brain tumor. Eur I Hum Genet 2006; 14: 561-566.

48 Muller A, Schackert HK, Lange B et al: A novel MSH2 germline mutation in homozygous state in two brothers with colorectal cancers diagnosed at the age of 11 and 12 years. Am J Med Genet $A$ 2006; 140: 195 - 199 .

49 Edelmann L, Edelmann W: Loss of DNA mismatch repair function and cancer predisposition in the mouse: animal models for human hereditary nonpolyposis colorectal cancer. Am J Med Genet C Semin Med Genet 2004; 129: 91-99.

50 Stumpf DA, Alksne JF, Annegers JF et al: Neurofibromatisis: conference statement. Arch Neurol 1988; 45: 575-578.

51 Landau M, Krafchik BR: The diagnostic value of cafe-au-lait macules. J Am Acad Dermatol 1999; 40: 877-890.

52 Wang Q, Montmain G, Ruano E et al: Neurofibromatosis type 1 gene as a mutational target in a mismatch repair-deficient cell type. Hum Genet 2003; 112: 117-123. 